\title{
Residual stresses in IN 718 Turbine Disks
}

\author{
C. Krempaszky ${ }^{1}$, E.A. Werner ${ }^{1}$, M. Stockinger ${ }^{2}$ \\ ${ }^{1}$ Lehrstuhl für Werkstoffkunde und Werkstoffmechanik and Christian-Doppler Laboratory, \\ TU München, Germany \\ ${ }^{2}$ Böhler Schmiedetechnik GmbH \& CoKG, Kapfenberg, Austria
}

Keywords: Residual stress, Quenching, Machining, Distortion.

\begin{abstract}
A semianalytical model is proposed to investigate the development of the residual stress in plate-like components and the resulting distortion during machining. The model is tested by comparison to fully numerical approaches and to experimental results.
\end{abstract}

\section{Introduction}

To obtain materials/components covering a wide range of conditions with characteristic mechanical properties, quenchings in connection with special heat treatments are of particular engineering importance. In addition to strength demands, a reduction of the production costs of forged parts by eliminating processing steps is essential. In this course a direct age version of IN 718 has been proposed for which an increase of strength is possible by precipitation strengthening during cooling from the forging temperature. The mechanical and thermal treatments inevitably result in residual stresses and distortions in the treated parts, which result from coupled thermo-mechanical interactions during the usually rapid cooling from sufficiently high temperatures down to room temperature [1,2]. Due to the macroscopic portion of the residual stress state, machining of slender forged components may lead to severe distortion of the component. In the worst case, the dimensional accuracy of the pre-finished component is not achieved.

Within the scope of this paper, the residual stresses in forged plate-like components are investigated with the focus on the distortion of the component during machining. To predict the transient thermal stresses during quenching and the resulting residual stress state a semi-analytical plate model is set up following the ideas of LANDAU and WEINER [3]. Based on these results the redistribution of the residual stresses and the corresponding distortion due to machining are estimated. Additionally, an approximative solution of the plate approach is proposed using the finite element method. To verify the theoretical predictions of the residual stress state and the corresponding distortion resulting from machining, the component distortion is determined by experiment considering turbine disks of the Ni-base superalloy IN 718.

\section{Semianalytic plate model}

The model of an infinite homogeneous isotropic plate is well suited for a qualitative study of the thermally induced residual stresses in plate-like components during cooling. The plate is 
cooled down symmetrically with respect to its middle plane. Because of the small strains occurring during quenching, the heat conduction problem and the thermomechanical analysis of quenching are sequentially coupled, i.e. the thermomechanical problem has no influence on the heat conduction problem. Details of this approach can be found in the literature $[3,4,5]$. Due to the symmetry to the middle plane of the plate the heat conduction problem and the thermomechanical analysis of quenching can be reduced to the half plate thickness. The constitutive behavior of the material is assumed to be elastic perfect plastic with a temperature dependent yield limit. All other material properties are assumed to be temperature independent. It can be shown, that during quenching the viscous part of the material behavior has a much smaller effect on the residual stress distribution than the dependence of the yield stress on the temperature [3]. An enhancement of the semianalytical approach to a more sophisticated constitutive behavior is a straight forward procedure. However, the resulting sets of equations become much more complex. Therefore an approximation using the finite element method is more efficient. Using the ABAQUS code, an approximation solution of the plate model is proposed in the next section. In the following, the basic assumptions of the plate model are outlined.

\section{Heat conduction}

The plate considered (constant thickness $2 R$, initial temperature $T_{1}$, thermal conductivity $\lambda$, thermal diffusivity $K$ ) is cooled down by free convection on both surfaces (time $\tilde{t}$, surface heat-transfer coefficient $a$ ) to environmental temperature $T_{0}$.

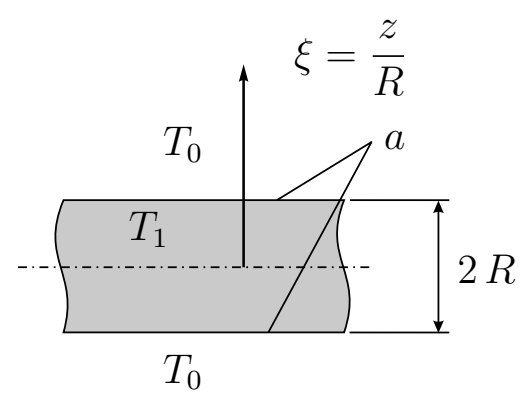

Figure 1: Plate model: symmetrical cooling

To formulate the heat conduction problem, the following non-dimensional quantities are introduced:

$$
\begin{aligned}
\mathrm{Bi} & =\frac{a R}{\lambda}, \\
\vartheta & =\frac{T-T_{0}}{T_{1}-T_{0}} \\
\xi & =\frac{z}{R}, \\
t & =\frac{K \tilde{t}}{R^{2}} .
\end{aligned}
$$

The Biot-Number Bi characterizes the surface heat transfer. The non-dimensional temperature $\vartheta$ as well as the spatial coordinate $\xi$ take values between 0 and $1(\xi=0$ is the midplane of the plate, $\xi=1$ is the surface). The FouriER-Number $t$ represents a dimensionless time coordinate. The one-dimensional heat conduction problem is characterized by 
the heat conduction equation

$$
\vartheta^{\prime \prime}-\ddot{\vartheta}=0
$$

and the initial and boundary conditions are

$$
\begin{aligned}
\vartheta(\xi, t=0) & =1, \\
\vartheta^{\prime}(\xi=0, t) & =0, \\
\vartheta^{\prime}(\xi=1, t) & =-\operatorname{Bi} \vartheta(\xi=1, t),
\end{aligned}
$$

where the dash and the dot denote the partial derivatives with respect to $\xi$ and $t$, respectively. The solution of (5) can be written as [7]:

$$
\vartheta(\xi, t)=\sum_{k}\left[A_{k} \cos \left(\delta_{k} \xi\right)+B_{k} \sin \left(\delta_{k} \xi\right)\right] e^{-\delta_{k}^{2} t}
$$

The initial and boundary conditions yield

$$
\begin{aligned}
A_{k} & =\frac{2 \sin \left(\delta_{k}\right)}{\delta_{k}+\sin \left(\delta_{k}\right) \cos \left(\delta_{k}\right)}, \\
B_{k} & =0,
\end{aligned}
$$

and the conditional equation for eigenvalues $\delta_{k}$,

$$
\delta_{k} \tan \left(\delta_{k}\right)=\mathrm{Bi}
$$

An estimation of the temperature distribution considering the partial sum of the first 20 terms of (6) is sufficient for the subsequent thermomechanical analysis.

Thermomechanical analysis of quenching

Considering a homogeneous isotropic constitutive behavior, a temperature distribution varying only in thickness direction $\xi$ leads to an equal biaxial stress distribution homogeneous with respect to the middle plane of the plate, i.e.

$$
\sigma_{11}=\sigma_{22}=\sigma ; \quad \sigma_{33}=\sigma_{12}=\sigma_{13}=\sigma_{23}=0,
$$

and a strain distribution also homogeneous and equal biaxial, i.e.

$$
\varepsilon_{11}=\varepsilon_{22}=\varepsilon ; \quad \varepsilon_{12}=\varepsilon_{13}=\varepsilon_{23}=0 .
$$

If we introduce non-dimensional stress and strain variables according to

$$
\begin{aligned}
s & =\frac{(1-\nu) \sigma}{E \alpha\left(T_{1}-T_{0}\right)}, \\
e & =\frac{\varepsilon}{\alpha\left(T_{1}-T_{0}\right)}
\end{aligned}
$$

(Young's modulus $E$, Poisson's ratio $\nu$, thermal expansion coefficient $\alpha$ ), then the equilibrium conditions result in

$$
\begin{gathered}
\int_{-1}^{1} s(\zeta) \zeta d \zeta=0, \\
\int_{-1}^{1} s(\zeta) d \zeta=0
\end{gathered}
$$


and the conditions of compatibility yield a linear strain distribution in thickness direction, i.e.

$$
e(\xi, t)=C_{1}(t)+C_{2}(t) \xi
$$

in accordance with Kirchhoff's hypothesis. Due to the symmetry of the field variables to the mid-plane, the moment-equilibrium equation (12) is always satisfied, the integration range of the force-equilibrium (13) can be reduced to the half plate thickness and (14) yields $e(\xi, t)=e(t)$. Hence the total strain is constant, but its thermal, elastic and plastic parts vary across the plate thickness according to

$$
e(t)=e_{\mathrm{th}}(\xi, t)+e_{\mathrm{e}}(\xi, t)+e_{\mathrm{p}}(\xi, t) .
$$

Considering (2) and (11) the non-dimensional thermal strain is equal to the non-dimensional temperature, i.e.

$$
e_{\mathrm{th}}(\xi, t)=\vartheta(\xi, t),
$$

and the elastic-plastic constitutive law can be written as

$$
s(\xi, t)=e_{\mathrm{e}}(\xi, t)=e(t)-e_{\mathrm{p}}(\xi, t)-\vartheta(\xi, t) .
$$

The yield condition indicates plastic deformation, if

$$
s^{2}=s_{\mathrm{y}}^{2}, \quad s \frac{d s}{d t} \geq 0
$$

and elastic behavior, if

$$
s^{2}<s_{\mathrm{y}}^{2} \text { or } s^{2}=s_{\mathrm{y}}^{2}, \quad s \frac{d s}{d t}<0,
$$

where

$$
s_{\mathrm{y}}=\frac{(1-\nu)}{E \alpha\left(T_{1}-T_{0}\right)} Y
$$

denotes a non-dimensional yield stress and $Y$ is the yield stress in tension. We consider a yield stress linearly dependent on temperature, i.e.

$$
s_{\mathrm{y}}=s_{\mathrm{y}}(\vartheta)=s_{\mathrm{y} 0}+k \vartheta,
$$

where $k>-s_{\mathrm{y} 0}$ ensures positive values of the yield stress in tension within the temperature range.

The range $0 \leq \xi \leq 1$ is split up into different regions, exhibiting elastic loading, plastic flow or elastic unloading. The formation of plastic regions and the onset of unloading is determined by Eqs. (18) and (19), respectively. The locations of the boundaries between these regions, $\xi_{\mathrm{i}}$, represent state variables characterizing the instantaneous stress distribution. At the interface between elastic and plastic regions two cases have to be distinguished:

- The plastic region is growing, i.e. the yield stress is reached at the interface $\xi_{\mathrm{i}}$

$$
e(t)-e_{\mathrm{p}}\left(\xi_{\mathrm{i}}, t\right)-\vartheta\left(\xi_{\mathrm{i}}, t\right)= \pm s_{\mathrm{y}}\left(\vartheta\left(\xi_{\mathrm{i}}, t\right)\right),
$$

(yielding in tension: upper sign; yielding in compression: lower sign) and the partial time derivative of the plastic strain is vanishing, i.e.

$$
\dot{e}_{\mathrm{p}}(\xi, t)=0 .
$$

On account of (21) and (23) the total time derivative of (22) leads to the evolution equation

$$
\dot{\xi}_{\mathrm{i}}(t)=\frac{\dot{e}(t)-(1 \pm k) \dot{\vartheta}\left(\xi_{\mathrm{i}}, t\right)}{e_{\mathrm{p}}^{\prime}\left(\xi_{\mathrm{i}}, t\right)+(1 \pm k) \vartheta^{\prime}\left(\xi_{\mathrm{i}}, t\right)} .
$$


- The elastic region is growing. In this case elastic unloading is starting at the interface, i.e.

$$
\dot{e}_{\mathrm{p}}\left(\xi_{\mathrm{i}}, t\right)=\dot{e}(t)-\dot{\vartheta}\left(\xi_{\mathrm{i}}, t\right) \mp \dot{s}_{\mathrm{y}}\left(\vartheta\left(\xi_{\mathrm{i}}, t\right)\right)=0
$$

Considering (21) the total derivative of (25) leads to the evolution equation

$$
\dot{\xi}_{\mathrm{i}}(t)=\frac{\ddot{e}(t)-(1 \pm k) \ddot{\vartheta}\left(\xi_{\mathrm{i}}, t\right)}{(1 \pm k) \dot{\vartheta}^{\prime}\left(\xi_{\mathrm{i}}, t\right)} .
$$

The evolution equations (24) or (26) respectively at the interfaces and the total time derivative of the force equilibrium (13) constitute a system of ordinary differential equations for the evolution of the total strain, $e(t)$ and the locations of the interfaces, $\xi_{\mathrm{i}}(t)$. The initial condition is $e_{\mathrm{p}}(\xi, 0)=0$.

$\underline{\text { Mechanical analysis of distortion during machining }}$

The material removal during machining is considered as a uniform thickness reduction of the plate. Thus, a new free surface is created and is characterized by the coordinate $\xi=\gamma$ as shown in Fig. 2. Additional residual stresses induced by the cutting process itself are neglected.

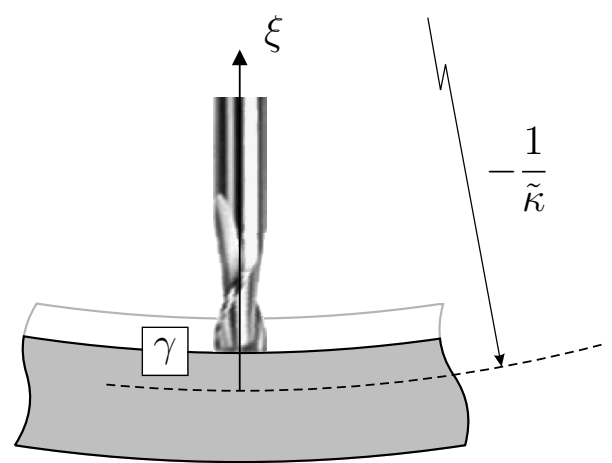

Figure 2: Plate model: layer removal

The thickness of the plate is reduced to the domain $-1 \leq \xi \leq \gamma$ and the equations of equilibrium of the machined plate become

$$
\int_{-1}^{\gamma} s(\xi) d \xi=0 ; \int_{-1}^{\gamma} s(\xi) \xi d \xi=0
$$

For sake of simplicity we assume a linear elastic constitutive law to model the stress redistribution. Thus the stress distribution of the distorted plate can be obtained by superposition of the residual stresses before machining,

$$
s_{\mathrm{r}}(\xi)=\lim _{t \rightarrow \infty}\left(e(t)-\vartheta(\xi, t)-e_{\mathrm{p}}(\xi, t)\right)=e^{\star}-e_{\mathrm{p}}^{\star}(\xi)
$$

and the change of stresses due to distortion $s_{\mathrm{d}}(\xi, \gamma)$. As a result of the material removal the symmetry of the residual stress state is lost leading to a curvature $\kappa_{\mathrm{d}}$ and an in-plane strain $\tilde{e}_{\mathrm{d}}$. The linear elastic constitutive law and the conditions of compatibility of the machined plate yield

$$
s_{\mathrm{d}}(\xi, \gamma)=e_{\mathrm{d}}(\xi, \gamma)=C_{1}(\gamma)+C_{2}(\gamma) \xi=\tilde{e}_{\mathrm{d}}(\gamma)-\kappa_{\mathrm{d}}(\gamma) \xi
$$


Hence the redistributed residual stresses can be written as

$$
s(\xi, \gamma)=s_{\mathrm{d}}(\xi, \gamma)+s_{\mathrm{r}}(\xi)=e_{\mathrm{d}}(\gamma)-\kappa_{\mathrm{d}}(\gamma) \xi-e_{\mathrm{p}}^{\star}(\xi)
$$

where $e_{\mathrm{d}}(\gamma)=e^{\star}+\tilde{e}_{\mathrm{d}}(\gamma)$. Taking into account the symmetric residual stress distributions estimated by the quenching model outlined above, the following initial values are obtained:

$$
e_{\mathrm{d}}(1)=e^{\star}=\frac{1}{2} \int_{-1}^{1} e_{\mathrm{p}}^{\star}(\xi) d \xi \text { and } \kappa_{\mathrm{d}}(1)=0 .
$$

The equilibrium conditions (27) and the stress distribution (30) lead to the relationships for the curvature and the in-plane strain in dependence of thickness of the removed layer $1-\gamma$ according to

$$
\begin{aligned}
& e_{\mathrm{d}}(\gamma)=4 \frac{\gamma^{2}-\gamma+1}{(\gamma+1)^{3}} \int_{-1}^{\gamma} e_{\mathrm{p}}^{\star}(\xi) d \xi+6 \frac{1-\gamma}{(\gamma+1)^{3}} \int_{-1}^{\gamma} e_{\mathrm{p}}^{\star}(\xi) \xi d \xi, \\
& \kappa_{\mathrm{d}}(\gamma)=6 \frac{\gamma-1}{(\gamma+1)^{3}} \int_{-1}^{\gamma} e_{\mathrm{p}}^{\star}(\xi) d \xi-\frac{12}{(\gamma+1)^{3}} \int_{-1}^{\gamma} e_{\mathrm{p}}^{\star}(\xi) \xi d \xi .
\end{aligned}
$$

Equations (31), (32) lead to the system of ordinary differential equations

$$
\begin{aligned}
0 & =\frac{d e_{\mathrm{d}}(\gamma)}{d \gamma}+\frac{2-\gamma}{3} \frac{d \kappa_{\mathrm{d}}(\gamma)}{d \gamma} \\
e_{\mathrm{p}}^{\star}(\xi=\gamma) & =e_{\mathrm{d}}(\gamma)-\gamma \kappa_{\mathrm{d}}(\gamma)-\left.\frac{(\gamma+1)^{2}}{6} \frac{d \kappa_{\mathrm{d}}}{d \gamma}\right|_{\gamma}
\end{aligned}
$$

from which the distributions of plastic strain and the associated residual stress from a known development of the distortion $e_{\mathrm{d}}(\gamma)$ and $\kappa_{\mathrm{d}}(\gamma)$, e.g. obtained from experiment, can be estimated.

\section{Numerical Approach}

Using the FE-code ABAQUS the plate model is realized by a row of thermally coupled axisymmetric elements (type CAX4T). The kinematic constraints to fulfill KIRCHHOFF's hypothesis are realized by appropriate symmetry and boundary conditions as shown in Fig. 3 using the SLIDE option.

thermally isolated; symmetry axis

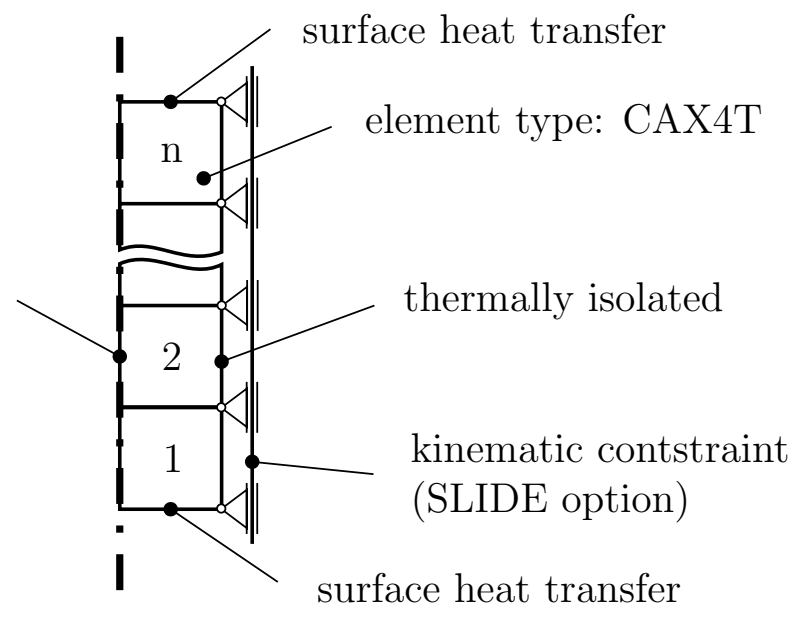

Figure 3: Plate model: finite element approach 
The machining process is realized by removing the elements successively. In this way the effects of a more sophisticated constitutive behavior e.g. viscosity, temperature dependencies of the material parameters can be easily evaluated to investigate the applicability of the semianalytic approach.

\section{Results and Discussion}

The residual stress distributions estimated by the semianalytical quenching model can be classified into 6 types as shown in Fig. 5:

- Type 0: No plastification, no resulting residual stresses

- Type 1: Plastification in tension at the border area starting at the surface, elastic unloading of the plastic zone, also starting at the surface

- Type 2: Plastification in tension at the border area and in compression in the middle area, elastic unloading of both zones

- Type 3: Same loading as in type 1, during unloading secondary plastification in compression of the border zone

- Type 4: Same loading as in type 2, during unloading secondary plastification in compression of the border zone

- Type 5: Same loading as in type 2, during unloading secondary plastification in compression of the border zone and secondary plastification in tension of the middle zone (cf. Fig. 4)

WeIneR and LANDAU $[5,3]$ identified 5 of them $(0,1,2,4,5)$ applying similar models. To verify the implementation of the governing equations, the results are compared to the finite element solution of the plate model showing excellent agreement (cf. Fig. 5). As an example the development of the interfaces between the different zones is shown in Fig. 4 for type 5. Considering the residual stress states of types 1 and 3 the plate is stress free, if the border zones are removed and no further distortion is to be expected. In contrast, residual stress states resulting from plastification of the inner region (i.e. cases 2, 4 and 5) are unfavorable, because they induce distortion during machining even though the border zones are already removed. Secondary plastifications lead to a drastic growth of the regions of high residual stress. In dependence of the characteristic variables $\mathrm{Bi}, s_{\mathrm{y}}$ and $k$ the type of residual stress state can be estimated as shown in Fig. 6. With increasing BioT-Number, Bi and decreasing yield limit, $s_{\mathrm{y}}$ the widths of the plastic zones occurring during quenching grow. In the limit case of $\mathrm{Bi} \rightarrow \infty$ and $s_{\mathrm{y}} \rightarrow 0$ the residual stress distribution is

$$
s=\left\{\begin{array}{cl}
-s_{\mathrm{y} 0} & \text { if } \quad 1 \geq|\xi|>0.5 \\
s_{\mathrm{y} 0} & \text { if } \quad 0.5 \geq|\xi| .
\end{array}\right.
$$

The redistribution of the residual stresses and the resulting curvature in dependence of the material removal is shown exemplarily in Fig. 7. In contrast to the simplifying assumption of elastic redistribution of the residual stresses during machining within the semianalytical approach, the material behavior is assumed to be elastic perfectly plastic in the finite element approach. This may lead to a slight underestimate of the curvature in the case of severe 
residual stresses (Fig. 7; right side). Figure 8 shows the curvature in dependence of the yield limit at fixed stages of material removal. It should be noted, that the ratio of curvature to yield limit tends asymptotically to a maximum value at a decreasing yield limit. Considering the limiting case of a rigid plastic plate (35), this maximum value can be estimated in dependence of the material removal specified by $\gamma$ as

$$
\frac{\kappa_{\max }}{s_{\mathrm{y} 0}}=\left\{\begin{array}{cll}
12 \frac{1-\gamma}{\left.(1+\gamma)^{3}\right)} & \text { if } \quad 0.5 \leq \gamma<1 \\
3 \frac{1+2 \gamma}{\left.(1+\gamma)^{3}\right)} & \text { if } \quad-0.5<\gamma<0.5 .
\end{array}\right.
$$

These upper bounds are plotted in Fig. 8.

The semianalytic plate model can also be applied to verify approximative solutions using e.g. the finite element method. Especially in the border regions high local mesh densities are required to approximate the high gradients of the field quantities. As an example, Fig. 9 shows some stress distributions in the middle of a thin circular plate during quenching estimated by a finite element model using the DEFORM2D ${ }^{\mathrm{TM}}$ code in comparison to the results of the plate model. Here 40 elements were used over the plate thickness and the FE results show a good correspondence to the semianalytical approach, thereby confirming the capability of DEFORM2D ${ }^{\mathrm{TM}}$ to predict the development plastic strain and residual stress.

Figure 10 shows a comparison of the predictions of the plate model to experimental results considering a turbine disk of the Ni-base alloy IN 718 (thickness approx. $20 \mathrm{~mm}$, diameter $320 \mathrm{~mm}$ ) forged at about $990^{\circ} \mathrm{C}$ and then water quenched. The disk was faced stepwise and the distortion of the backside was recorded simultaneously using a specially instrumented CNC lathe. Details of the experimental setup will be published elsewhere. The distortion is quite well approximated by a rough estimate using the plate model assuming a BIOT-Number $2<\mathrm{Bi}<3$. This corresponds to a heat transfer coefficient of $a=2000-3000 \mathrm{~W} / \mathrm{m}^{2} \mathrm{~K}$ and is representative for water quenching [6].

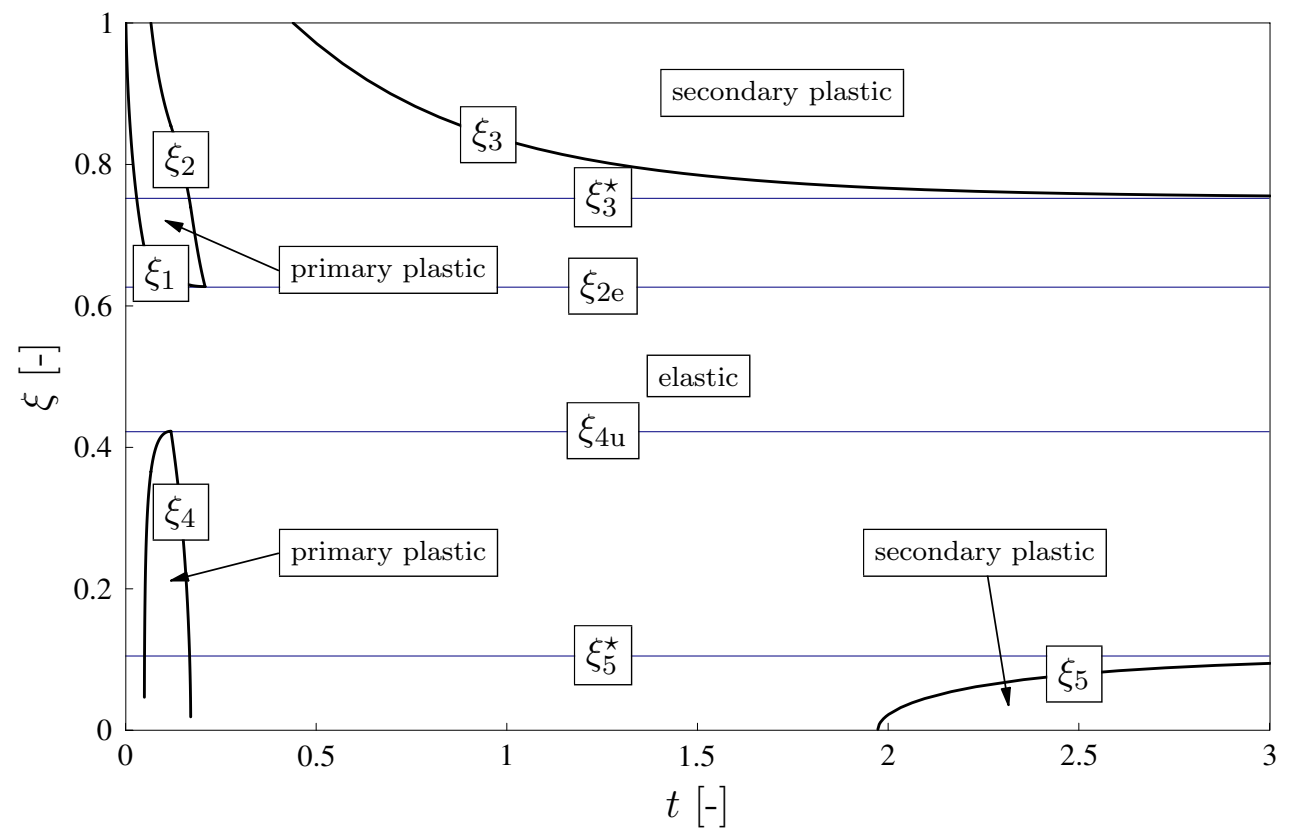

Figure 4: Development of the interfaces $\xi_{i}$ between the elastic and plastic regions (type 5) 

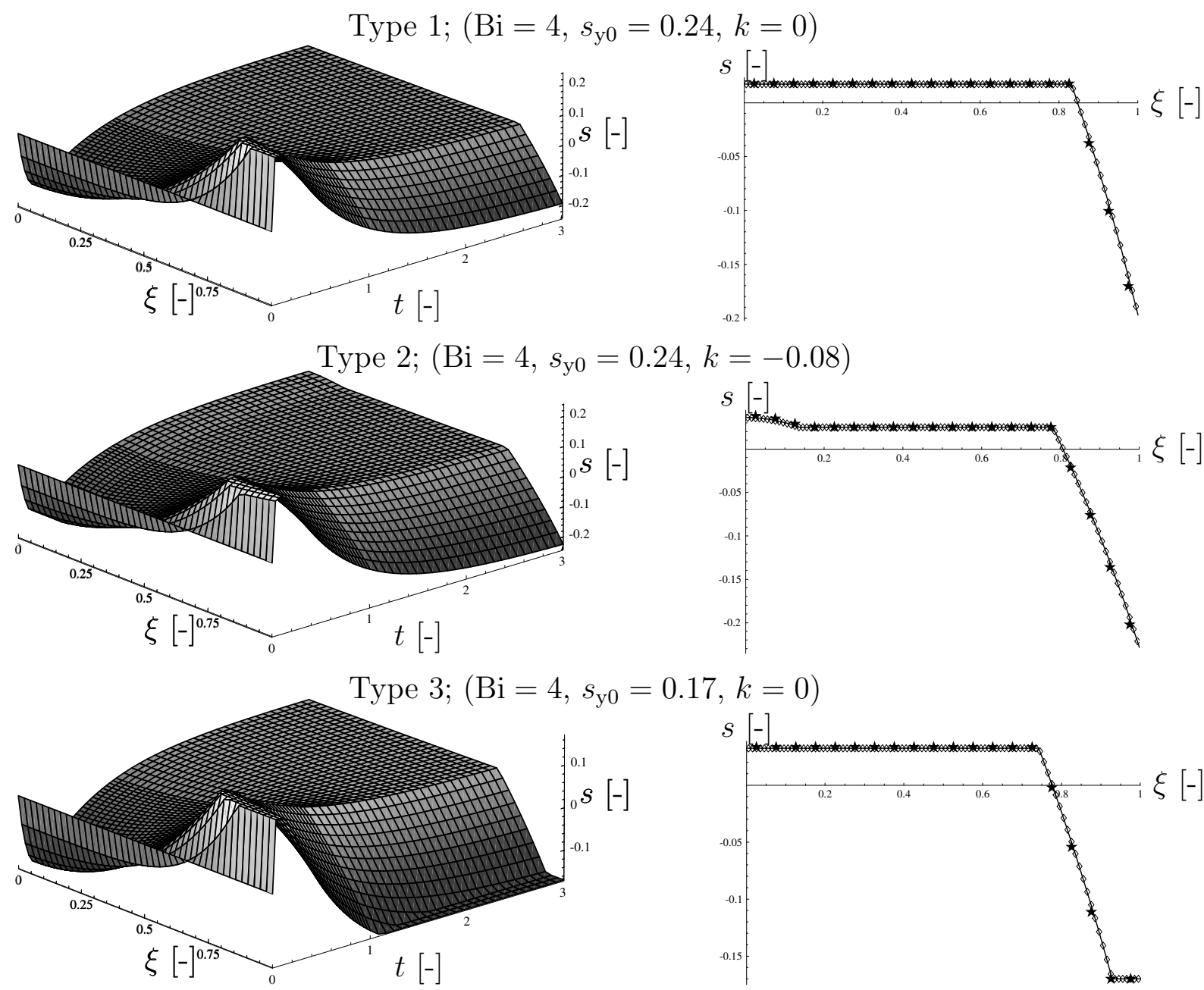

Type $4 ;\left(\mathrm{Bi}=4, s_{\mathrm{y} 0}=0.24, k=-0.08\right)$
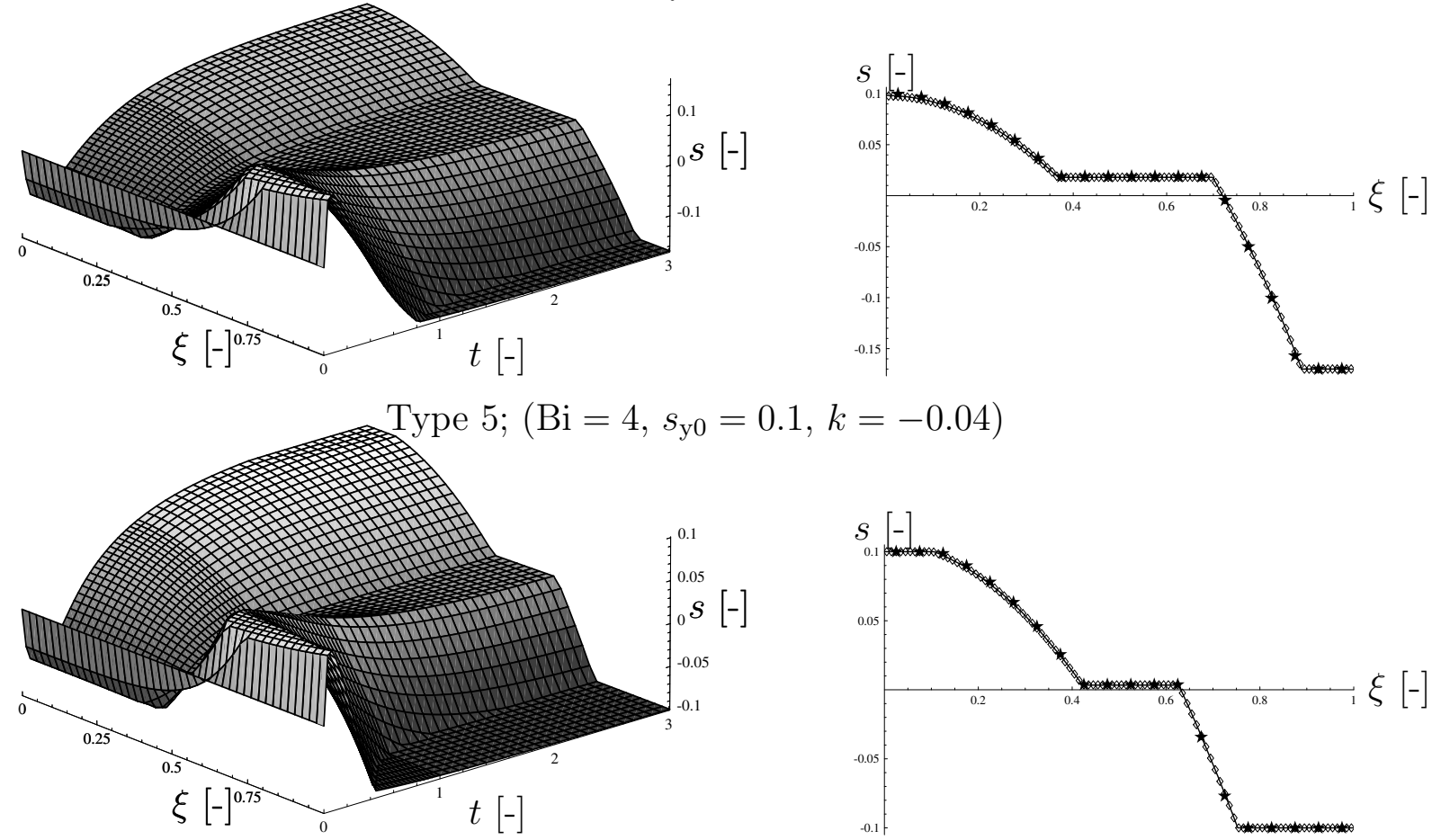

Figure 5: Classification of residual stress distributions: Development during quenching and residual stress state (line: semianalytical approach; star and diamond: finite element approach, different discretizations) 

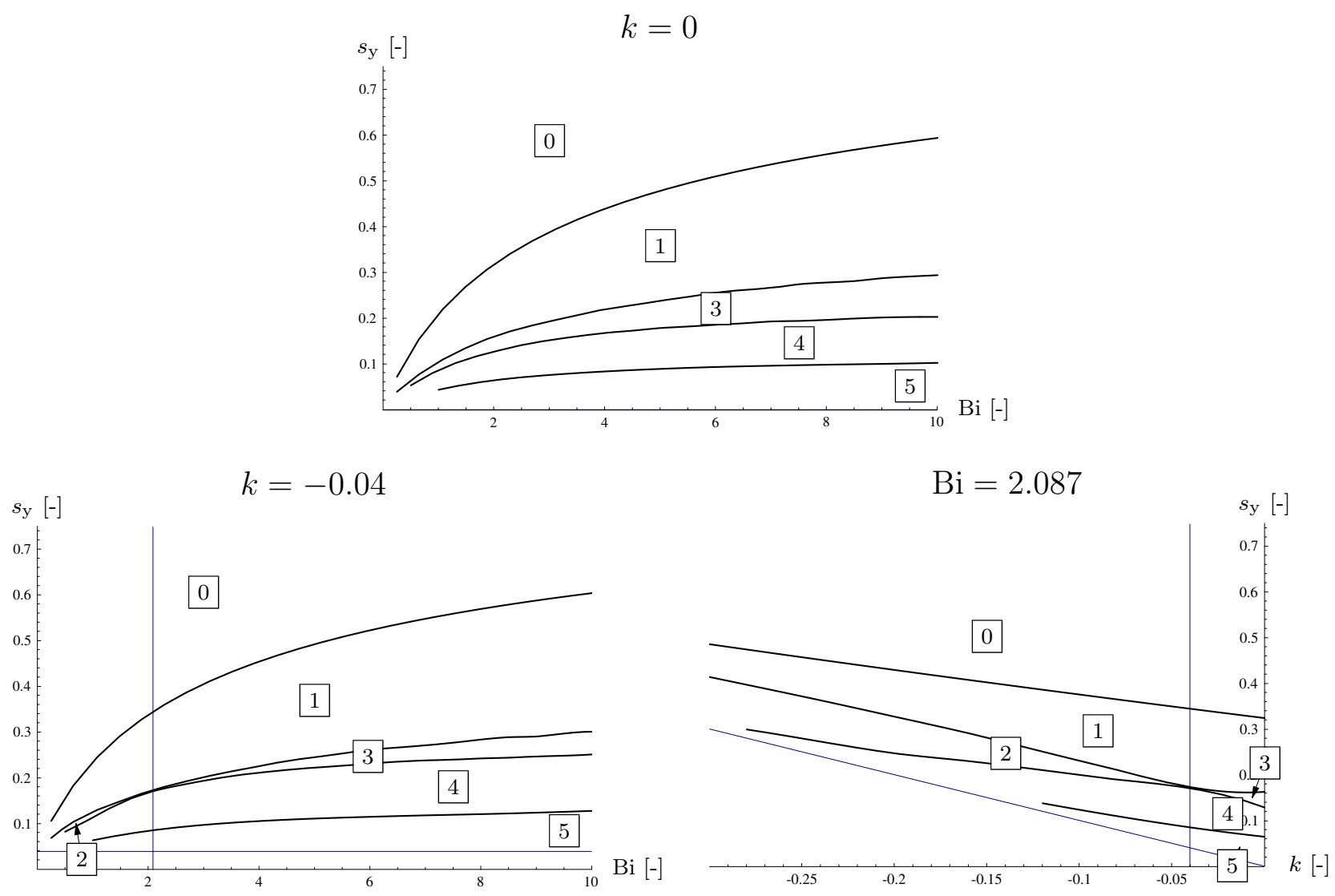

Figure 6: Types of residual stress state in dependence of $\mathrm{Bi}, s_{\mathrm{y}}$ and $k$

Type $3 ;\left(\mathrm{Bi}=4, s_{\mathrm{y} 0}=.2, k=0\right)$
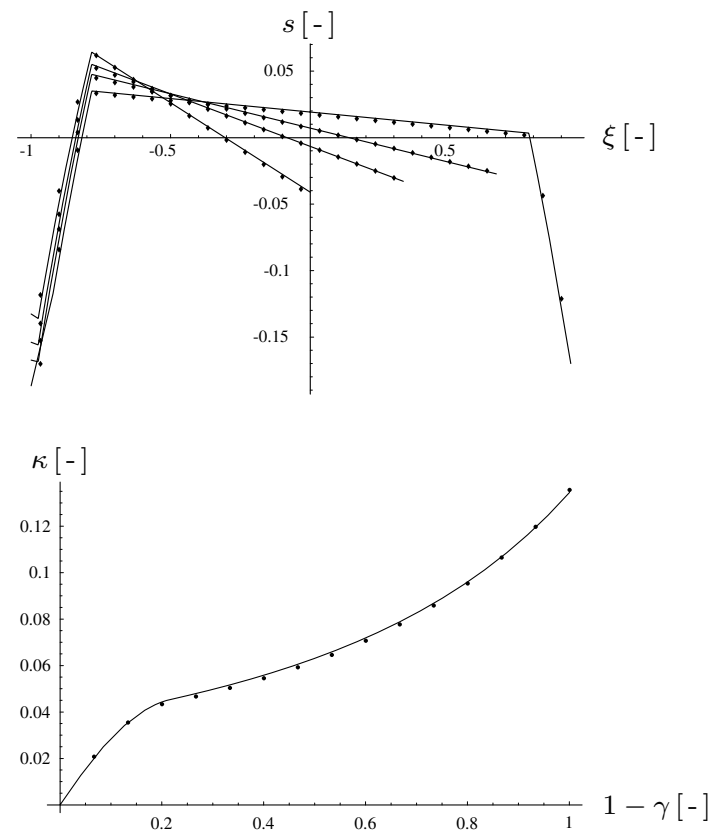

Type 5; $\left(\mathrm{Bi}=4, s_{\mathrm{y} 0}=.08, k=0\right)$
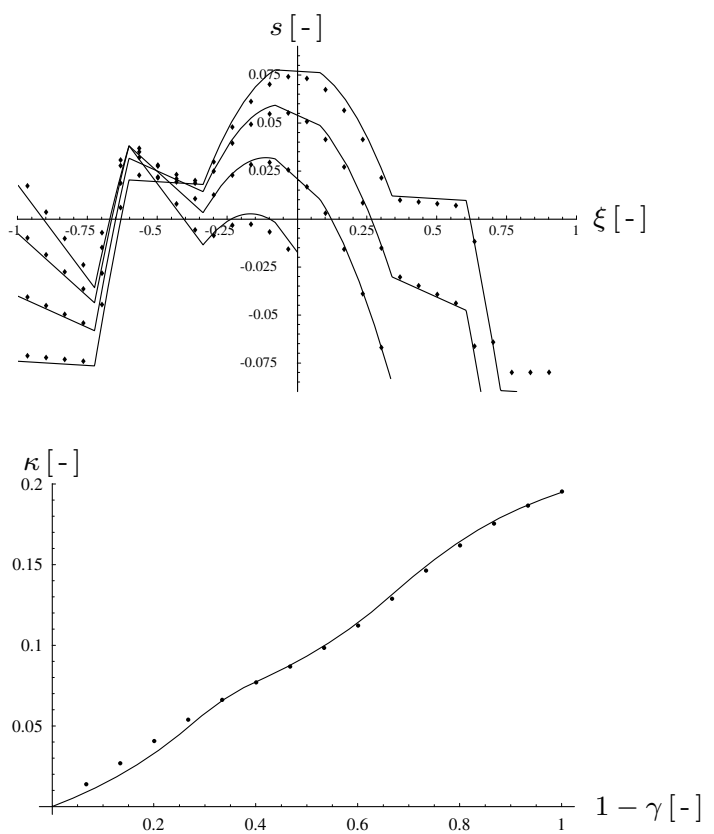

Figure 7: Redistribution of the residual stresses and development of the curvature in dependence of the material removal; line: semianalytical approach; dot: finite element approach 


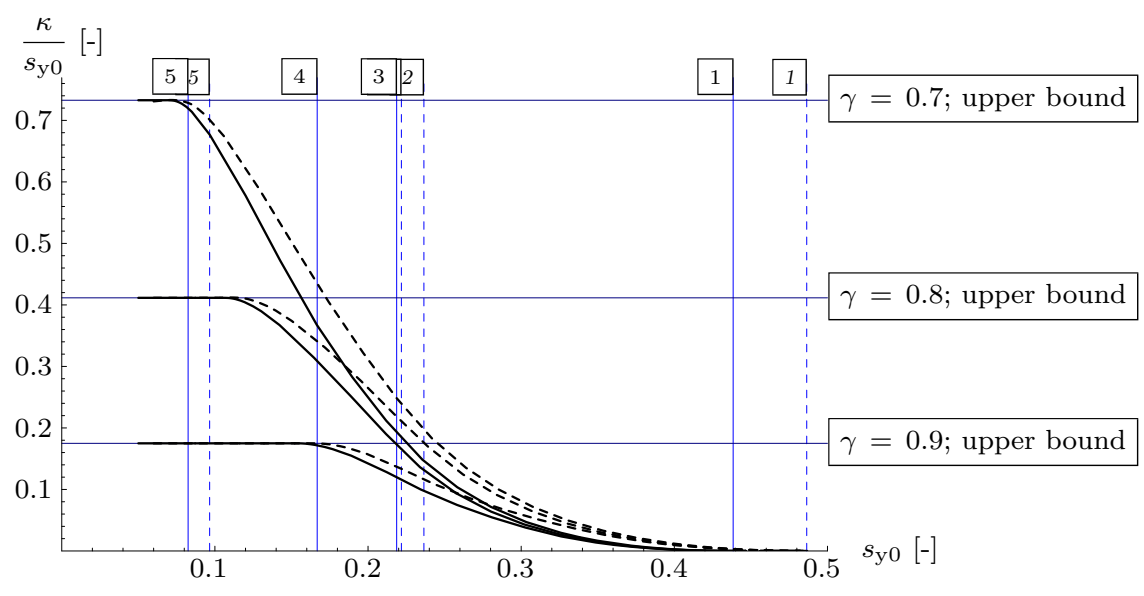

Figure 8: Ratio of curvature to yield limit in dependence of the yield limit and its upper bound estimated by (36) at three levels of material removal $(\gamma=0.9, \gamma=0.8$ and $\gamma=0.7)$; $\mathrm{Bi}=4$; solid lines: $k=0$, dashed lines: $k / s_{\mathrm{y} 0}=-0.25$

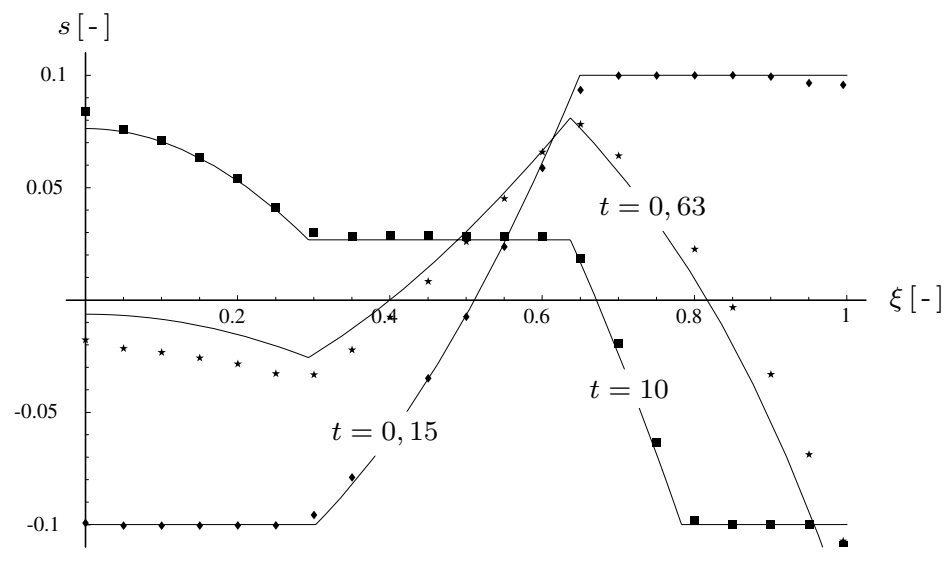

Figure 9: Development of the stress distribution $\mathrm{Bi}=4, s_{\mathrm{y} 0}=0,1, k=0$; dots: DEFORM2D ${ }^{\mathrm{TM}}$; lines: plate model

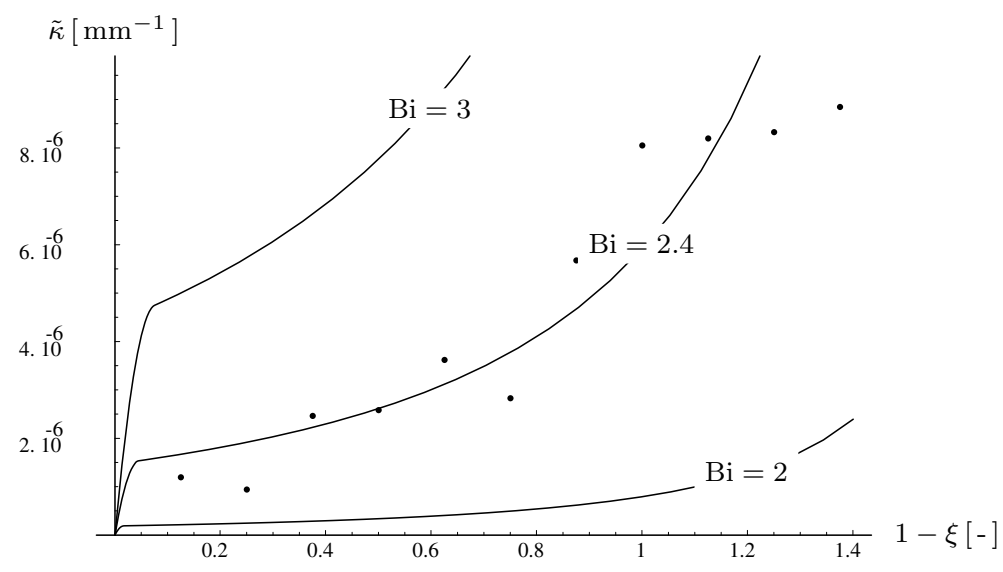

Figure 10: Curvature of a turbine disk in dependence of the material removal; Experiment (dots) and plate model (curves) 


\section{Summary}

The proposed semianalytic plate model is a fast and simple tool for a qualitative study of residual stresses induced by quenching as well as their redistribution and the resulting distortion of plate-like component during machining. Five types of residual stress states induced by quenching can be classified and mapped in dependence of three characteristic variables, the Biot-number, the normalized yield stress and the reduction of the yield stress due to temperature rise. The semianalytical solutions can also help to analyze the required discretization of finite element based models of the quenching problem.

\section{References}

[1] D. Dye, K. T. Conlon and R. C. Reed, "Characterization and Modeling of QuenchingInduced Residual Stresses in the Nickel-Based Superalloy IN 718," Metallurgical and Materials Transactions A., 35A (2004), 1703-1713.

[2] H. Fecht and D. Furrer, "Processing of Nickel-Base Superalloys for Turbine Engine Disc Applications," Advanced Engineering Materials, 12 (2000), 777-787.

[3] H. G. Landau, J. H. Weiner, and E. E. Zwicky. Thermal Stress in a Viscoelastic-Plastic Plate with Temperature-Dependent Yield Stress. J. Appl. Mech., 27:297-302, 1960.

[4] H. G. Landau and J. H. Weiner. Transient and residual stresses in heat treated plates. J. Appl. Mech., 80:459-465, 1958.

[5] J. H. Weiner. An elastoplastic thermal-stress analysis of a free plate. J. Appl. Mech., 78:397-401, 1956.

[6] B. Liščić, H. M. Tensi, W. Luty. Theory and Technology of Quenching. Springer Verlag Berlin, 1992.

[7] H. S. Carslaw, J. C. Jaeger. Conduction of Heat in Solids. Calendron Press Oxford, 1990. 